limited to putative synaptic transmitters and excludes myelin lipids, carbohydrates, electrolytes, and other 'chemicals' of the brain.

Most of the book is organized regionally, with chapters on the peripheral nervous system, spinal cord, retina, raphé nuclei of the brainstem, cerebellar cortex, thalamus, hypothalamus, hippocampus, amygdala, olfactory bulb, corpus striatum, and cerebral cortex. An exception is a chapter on catecholamine systems, a chemical rather than a strictly anatomical arrangement. The book is profusely illustrated and well indexed. Most chapters contain a list of references which are extensive enough to alone be worth the purchase price.

In addition to reviewing the large body of published literature, new information also is offered. For example, Steinbusch and Nieuwenhuys provide a new immunocytochemical method for the direct localization of serotonin in sections of brain and offer convincing photomicrographs and an atlas of the rat brainstem using this method. The chapter on the peripheral nervous system by Schultzberg is well done and discusses many newly discovered peptides in addition to the now classical data on monoamines and acetylcholine. The olfactory bulb is discussed by Macrides and Davis in relation to transmitters including substance $P$, opioid peptides, somatostatin, and others, in greater depth than I previously have seen.

I would have enjoyed reading more discussions of the chemical anatomy of the nervous systems of nonmammalian vertebrates and invertebrates for comparison with mammals, as was done by Brecha in his chapter on retinal transmitters. In general I would recommend this book to any neuroscientists investigating neurotransmitters. This critical survey will save many hours in the library integrating voluminous data from the original sources.

Harvey B. Sarnat, Calgary, Alberta

\section{CLINICAL AND BIOLOGICAL ASPECTS OF PERIPHERAL} NERVE DISEASES. Proceedings of the Symposium on Clinical and Biological Aspects of the Peripheral Nervous System Diseases held in Padova, Italy, September 9-12, 1982. Edited by L. Battistin, G.A. Hashim, and A. Lajtha. Published by Alan R. Liss, Inc., New York. 402 pages. $\$ 60 \mathrm{Cdn}$. approx.

This book documents the proceedings of a symposium held in Padova in September 1982, on peripheral nerve disorders. As might be expected, the majority of the papers are of interest to those working actively in the field rather than to clinical neurascientists, but this volume does contain some communications of more general interest. For example, there is a clinical overview which provides a concise summary of the major causes of peripheral neuropathy, their distinctions, and their management. There is a well referenced discussion of the electrophysiological findings in peripheral nerve diseases. There is a section on epidemiology, which includes a discussion of the relationship of the Guillian-Barre syndrome to immunization against "swine flu", and to other antecedent events. The immunological aspects of experimental and of human peripheral nerve diseases are well reviewed in another paper, and there is a summary of recent work on serum-induced demyelination. In another section there is an intriguing report on the recording of spontaneous activity, with microelectrodes from patients with "positive"' symptoms following peripheral nerve injuries. These and other papers may be useful for reference.

Peter Ashby,

Toronto, Ontario

PEDIATRIC HEAD TRAUMA. 1983. Edited by Kenneth Shapiro, M.D. Futura Publishing Company, Mount Kisco, New York. 296 pages. $\$ 46 \mathrm{Cdn}$. approx.

Those practitioners who care for children with head injury are often frustrated by the lack of reference to children's treatment in scientific reports on this topic. Thus, one hopes that this concise text will put the problem of pediatric head trauma in to perspective.

Right away, the first chapter must come to grips with the blending of statistics, while it considers the epidemiology of head trauma in children. The discussion refers to a patient population less than 14 years of age and in so doing, the author has had to extract figures from very large series which are all age inclusive. That done, the expected comes forth. More boys than girls suffer injuries, falls and road traffic accidents are by far the commonest culprits, and fortunately, children who have suffered one head injury, are not at increased risk of further insult. The medical, legal and ethical aspects of child abuse are considered in a separate chapter, and the warning signs of possible abuse enumerated. There is re-emphasis of the "Whiplash Shake Syndrome", which can be the covert explanation for subdural hematomata and more particularly unexplained retinal hemorrhages found in infants.

The initial triage decisions are next considered, first for children with head injury neither severe nor productive of lasting coma. It is calcualted that of a substantial number of children who are assessed for head injury, less than $10 \%$ are admitted to hospital for observation. The challenge is placed whether this "under-evaluation" and "inappropriate triage has the potential of leading to grave complications". So, appropriate guidelines are provided. The matter of routine skull radiography is in general up for grabs, but in this instance, guidelines are precise about the characteristics of fractures in children's skulls.

The fact that $8 \%$ of children seen at random in the emergency room with head injury have skull fractures, whereas $27 \%$ of those admitted for observation have same, perhaps underscores what has been suspect for some time - namely, that triage decisions can more reliably be made on the basis of history and clinical examination than abnormal skull radiography. In passing, one takes issue with the statement that epidural hematomata are capable in some instances of decompressing themselves through the overlying fracture.

The radiology chapter is quite thorough, a mini-text of its own. The author has resisted the temptation to speak only about CT diagnosis of head injury, and has wisely begun this section with details of skull and spine radiography, their usefulness and pitfalls. The experience is amply supported by literature references as well as personal case encounters from the author's own children's hospital. 
There is not much that the neurosurgeon can do about the "first injury", so that one's therapeutic impact focuses on the prevention of the "second injury". The latter can be produced by hypoxia and hypercarbia, themselves having a myriad of causes. The prevention of the "second injury" is the theme of the well-written chapter on the care for the child with the "severe" head injury. The reader is taken step-wise through the child's management from the scene, to the Emergency Room and then beyond. Orthodox principles are again enunciated as they must so often be. We learn from this particular author's broad experience that the Glasgow Coma Scale while not ideal has its purposes. Thus, patients with GCS of 7 or less are intubated, those at 5 or below have an ICP monitor placed and ventilation controlled. Corticosteroids are used in children with scores of 5 or less, anticonvulsants and antibiotics not "routinely adminstered" and barbiturates held for patients who show spontaneous waves of pressure or baseline ICP above 20 torr.

This chapter is followed by one which deals with the mechanisms and treatment of intracranial hypertension in children. Basic principles are again stated, and amply illustrated with the results of experimental work. The only fault with this section may be its sequencing; it would have better served as a lead-in to the preceding chapter.

The section on the neuropathology of children's head injuries is written in exquisite detail; for example, brain edema following a non-infected penetrating wound is "present already 4 minutes after injury, and within 30 minutes to 2 hours strikingly increases in severity and extent". The author makes every effort to confine his remarks to the injury in children, and the principals and literature sources which are referenced provide an excellent review of the pathology of head trauma. There is considerable emphasis on the biomechanics of injury, and the effects of shearing. Not satisfied with the direct cerebral consequences of trauma, there is reference to secondary insults to cranial nerves, pituitary and vascular structures, as well as the sequelae of raised ICP. While the chapter concludes with anticipated comments on the late sequelae of head injury and the pathology of the persistent vegetative state, its overall topicality is represented by several paragraphs on the pathology of the "respirator brain".

The experienced clinician who thus far is au fait with the facts presented in the text may simply pick it up to determine what outcome can be expected in a child with a severe head injury. The chapter devoted to this aspect could very well be the most controversial in the text. A number of pediatric neurosurgeons would take issue with the statement that a "6-10\% mortality is as high as should be expected in the absence of major multiple trauma", and that "children less than 15 years of age who survive from acute head trauma rarely are left in a chronic vegetative or severely disabled state". While there is a wideheld belief that children with severe head trauma "do better" than adults, a principal again set forth in this chapter, it may very well turn out that material accumulating in the International Data Bank Series will disprove this.

The author of a separate section on post-traumatic epilepsy in children notes that while early seizures may be an indicator of the injury severity, it "is unclear whether early seizures per se have any influence on ultimate prognosis". While it is acknowledged that such early seizures can only complicate the general management of head injured children, it is recognized that attempts to diminish the frequency of early seizures can be very awkward. Late seizures are more ominous, often reflecting the severity of the original head injury. As a single late post-traumatic seizure carries a recurrence risk of $45 \%$, therapy is recommended, beginning with phenytoin, and it is again stressed that single drug administration is preferred to "polytherapy".

Those fortunate to have a children's rehabilitation facility available for their patient's further care will appreciate the detailed planning outlined in a chapter on this topic. Such therapy is multi-disciplined, must begin while the child is still in the acute phase of recovery and is dedicated to restoration of function to the highest level permitted by the organic deficit(s).

When the litigation process begins after a child's injury, the reporting physician desperately needs guidance on the expected neuropsychologic outcome for his patient. A chapter on this topic reminds us that the biologic "plasticity" of the child's brain (after injury) may not be what we think it is and thus children are not relatively impervious to congitive impairment after closed head injury. In fact, memory and motor skills may be substantially impaired. The wisdom imparted here by experienced authorities is worth reviewing before preparing the next medico-legal report.

All the contributors to this book dedicated themselves to the task of reporting on children's head injuries and they have in my view succeeded. Every now and then there creeps in acknowledgement of adult experience, but the volume is a worthy purchase because of its adherence to the stated theme, and in particular for certain excellent chapters.

Robin P. Humphreys, Toronto, Ontario

\section{Books Received}

CATECHOLAMINES AND BEHAVIOUR. 1984. By Stephen

T. Mason. Published by Cambridge University Press. 464 pages. $\$ 74 \mathrm{Cdn}$. approx. (hardcover), \$31 Cdn. approx. (paperback).

CATECHOLAMINES, PART A: BASIC AND PERIPHERAL MECHANISMS. Series: Neurology and Neurobiology. Edited by Earl Usdin, Arvid Carlsson, Annica Dahlstrom and Jorgen Engel. Published by Alan R. Liss. 420 pages. $\$ 150 \mathrm{Cdn}$. approx.
CATECHOLAMINES, PART B: NEUROPHARMACOLOGY AND CENTRAL NERVOUS SYSTEM - THEORETICAL ASPECTS. Series: Neurology and Neurobiology. Edited by Earl Usdin, Arvid Carlsson, Annica Dahlstrom and Jorgen Engel. Published by Alan R. Liss. 520 pages. $\$ 175 \mathrm{Cdn}$. approx.

CATECHOLAMINES, PARTC: NEUROPHARMACOLOGY AND CENTRAL NERVOUS SYSTEM - THERAPEUTIC 